\title{
Caracterização imunofenotípica e avaliação quantitativa do infiltrado linfocítário junto às áreas de displasia epitelial da próstata de cães adultos sexualmente intactos
}

\author{
Immunophenotipic characterization and quantitative evaluation of lymphocitary infiltrate \\ in areas with prostatic epithelial dysplasia of intact and adult dogs
}

\author{
Cristiane Padrin CaldeiraI Giovana Wingeter Di Santis ${ }^{\mathrm{II}}$ Júlio César Carvalho Balieiro ${ }^{\mathrm{III}}$ \\ Renée Laufer Amorim ${ }^{\mathrm{IV}}$ Veridiana Maria Brianezi Dignani de Mourav
}

\section{RESUMO}

O estudo da próstata canina tem se tornado comum em razão da grande incidência de doenças prostáticas nessa espécie e das similaridades com as alterações apresentadas pela glândula prostática humana. Frente à alta frequência de displasias epiteliais acompanhadas de infiltrado linfocitário intersticial e atrofia acinar na espécie canina, o presente estudo teve como objetivos a caracterização imunofenotípica e a avaliação quantitativa desse infiltrado, utilizando marcadores para identificação de linfócitos $T$ (antiCD3) e B (anti-CD79a). Foram catalogadas 42 lesões displásicas classificadas em discreta (48\%), moderada (38\%) e acentuada (14\%). O infiltrado linfocitário intersticial periacinar junto às áreas de epitélio prostático displásico constituiu-se predominantemente por linfócitos $T(66 \%) e$ houve interação entre o grau histológico da displasia e o marcador imunoistoquímico, com oscilação na quantidade de células $T$ e $B$ intersticiais em função do grau da displasia epitelial.

Palavras-chave: cão, linfócito $T$, linfócito $B, C D 3, C D 79 a$.

\section{ABSTRACT}

Canine prostatic studies have been common due to high incidence of prostatic diseases in these animals and similarities with alterations in human prostatic gland. Due the high frequency of dysplasia associated with interstitial lymphocitary infiltrate and acinar atrophy in canine prostate, the aims in this study were the immunophenotypic characterization and the quantitative evaluation of the same infiltrated using anti-CD3 and anti-CD79a to $T$ and $B$ lymphocytes, respectively. Forty two epithelial dysplasic lesions were graduated in discrete (48\%), moderate (38\%) and accentuated (14\%). Lymphocitary periacinar infiltrate in dysplasic areas was $T$ type and interaction between dysplasia grade and marker was observed, with oscillation of $T$ and $B$ cells in according with epithelial dysplasia grade.

Key words: dog, T lymphocyte, B lymphocyte, CD3, CD79a.

\section{INTRODUÇÃO}

O controle do crescimento prostático é influenciado por fatores intrínsecos e extrínsecos, entre esses, a presença de infiltrado inflamatório não relacionado a micro-organismos é considerada fator somático para o desenvolvimento de alterações prostáticas, visto que essas células podem produzir interleucinas, estimular a formação de matriz pela síntese de colágeno e promover a proliferação das células estromais e epiteliais (LEE et al., 1997).

Considerando a interação entre o epitélio e o estroma no desenvolvimento das alterações prostáticas, ORTIZ et al. (1996) notaram relação significativa entre displasia epitelial acentuada e infiltrado inflamatório mononuclear intersticial; entretanto, o tipo ou a intensidade do infiltrado, assim como sua proximidade aos focos da lesão, não foram avaliados. Também DE MOURA (2004) observou alta incidência de displasia epitelial acompanhada de infiltrado inflamatório intersticial periacinar na próstata de cães. Já MAHAPOKAI et al. (2001) utilizaram

ICurso de Medicina Veterinária, Centro Universitário Fundação de Ensino Octávio Bastos (UNIFEOB), São João da Boa Vista, SP, Brasil.

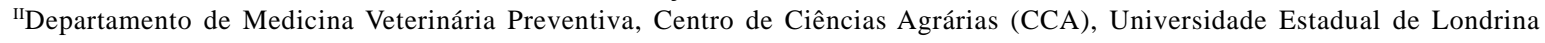
(UEL), Londrina, PR, Brasil.

IIIDepartamento de Ciências Básicas, Faculdade de Zootecnia e Engenharia de Alimentos (FZEA), Universidade de São Paulo (USP), Pirassununga, SP, Brasil.

Iv Serviço de Patologia Veterinária, Departamento de Clínica Veterinária, Faculdade de Medicina Veterinária e Zootecnia (FMVZ), Universidade Estadual Paulista (UNESP), Botucatu, SP, Brasil.

'Setor de Patologia Animal, Departamento de Medicina Veterinária, Escola de Veterinária (EV), Universidade Federal de Goiás (UFG), Estrada do Campus, s/nº, Campus II, 74001-970, CP 131, Goiânia, GO, Brasil. E-mail: vdmoura@vet.ufg.br. Autor para correspondência. 
marcadores imunoistoquímicos para identificar linfócitos T e B, macrófagos e plasmócitos no infiltrado inflamatório da próstata canina. Constataram que mais de $80 \%$ das células inflamatórias eram linfócitos T. Os demais tipos celulares estavam presentes, mas representaram frações menores do infiltrado. Os autores concluíram que a imunidade celular constitui o principal elemento envolvido na resposta inflamatória.

Apesar disso, a literatura é escassa no que diz respeito à displasia epitelial prostática na espécie canina. Contudo, a presença dessa alteração na próstata do cão suscita a necessidade de avaliar detalhadamente suas características histológicas, seu comportamento biológico e sua interação com as demais alterações do tecido prostático. Fundamentado em uma dessas interações, o presente estudo vislumbra alguma contribuição no esclarecimento da relação entre displasia e infiltrado inflamatório intersticial periacinar, já que tal concomitância é frequentemente observada entre as alterações da próstata canina (AMORIM, 2001; DE MOURA, 2004). Assim, os objetivos deste trabalho foram determinar o imunofenótipo T ou B dos linfócitos intersticiais adjacentes às áreas de displasia epitelial da próstata de cães adultos não castrados e quantificar os linfócitos T e B identificados.

\section{MATERIAL E MÉTODOS}

Foram estudadas 14 próstatas de cães com idade igual ou superior a quatro anos, intactos e com ou sem histórico de afecção prostática. As glândulas foram colhidas em exames necroscópicos e fixadas em formol tamponado a $10 \%$, durante 48 horas. Após a fixação, foram realizados seis cortes e obtidos os seguintes seguimentos prostáticos: cranial, medial e caudal, todos com lateralidade direita e esquerda. O material foi processado e incluído em parafina, e os cortes foram corados por Hematoxilina e Eosina (HE). As áreas displásicas foram caracterizadas a partir de critérios adaptados de BRASILEIRO FILHO et al. (2006) e DE MARZO et al. (2006). Consideraram-se áreas displásicas as compostas por ácinos de tamanhos e formas variáveis e formados por mais de uma camada de células epiteliais distintas, e estas apresentavam organização anormal e atipias, como citoplasma marcadamente reduzido e hipocromático, além de núcleo volumoso e nucléolo evidente. As alterações epiteliais displásicas foram classificadas em discreta, moderada e acentuada, de acordo com a intensidade das lesões, e eram sempre acompanhadas de infiltrado inflamatório mononuclear intersticial adjacente.

Na técnica de imunoistoquímica, cada anticorpo foi diluído em solução de BSA a 1\%. As concentrações testadas, otimizadas e empregadas foram 1:100 e 1:50, para anti-CD3 (pan-linfocitário T policlonal - DAKO A0452) e anti-CD79a clone HM57 (pan-linfocitário B monoclonal - DAKO M7051), respectivamente. Cortes de tecido prostático de $3 \mu \mathrm{m}$ foram distendidos sobre lâminas histológicas sinalizadas em poly-l-lysine (SIGMA P8920) e permaneceram em estufa durante 24 horas, mantendose a temperatura entre 55 e $58^{\circ} \mathrm{C}$. Em seguida, as lâminas foram diafanizadas e hidratadas.

A recuperação antigênica foi realizada em banho-maria, a $95^{\circ} \mathrm{C}$, com a solução tampão de EDTA pH 8,0. O bloqueio da peroxidase endógena foi realizado por incubação das lâminas em solução de água oxigenada 10 volumes. Em seguida, o material foi lavado em água destilada e em solução tampão TRIS pH 7,4. Foram incubados em câmara úmida, por 18 horas (overnight) e à temperatura de $4^{\circ} \mathrm{C}$, os cortes com os anticorpos primários. Na sequência, realizaram-se lavagem em TRIS pH 7,4 e incubação em anticorpo secundário (LSAB - DAKO K0690), em câmara úmida e à temperatura ambiente, conforme recomendação do fabricante.

Para a visualização da reação, utilizou-se solução de diaminobenzidina (DAB) (DAKO K3468), seguida de lavagem em solução tampão TRIS e água destilada. Em seguida, realizaram-se a contracoloração em Hematoxilina de Mayer, lavagem e desidratação. Na última etapa, realizaram-se a desidratação dos cortes e a montagem das lâminas com resina sintética (Permount - Fisher Scientific) e lamínulas histológicas.

O controle negativo para proteínas inespecíficas foi realizado substituindo-se o anticorpo primário por BSA a $1 \%$. Como controle negativo específico, substituiu-se o anticorpo primário monoclonal ou policlonal por imunoglobulinas produzidas em camundongo (DAKO N1698) e em coelho (DAKO X0903), respectivamente. Amostras teciduais de linfonodo humano foram utilizadas como controle positivo, já que ambos os anticorpos são produzidos para a utilização no tecido humano, mas apresentam reconhecida reatividade cruzada no tecido canino.

Para a quantificação das células linfoides marcadas para CD3 e CD79a junto às áreas de displasia, utilizou-se um microscópio óptico ligado a um equipamento de análise morfométrica de imagem, programa KS-300 (Zeiss). Para a contagem de uma área representativa, foram capturadas as imagens de quatro campos de cada lesão à objetiva de 40x, e contados os linfócitos positivos e negativos para cada anticorpo. A figura 1 exemplifica o método de contagem utilizado para quantificar o infiltrado inflamatório.

Para a avaliação das porcentagens de células marcadas segundo os diferentes graus histológicos de displasia e marcadores testados, utilizou-se o Método de Quadrados Mínimos Generalizados, pressupondo distribuição Binomial e função de ligação logística. As análises foram realizadas por meio do procedimento PROC GENMOD do programa Statistical Analysis System, versão 8.02 (SAS, 1995). Foram considerados os efeitos principais de grau (I - discreto, II - moderado e III - acentuado) e 


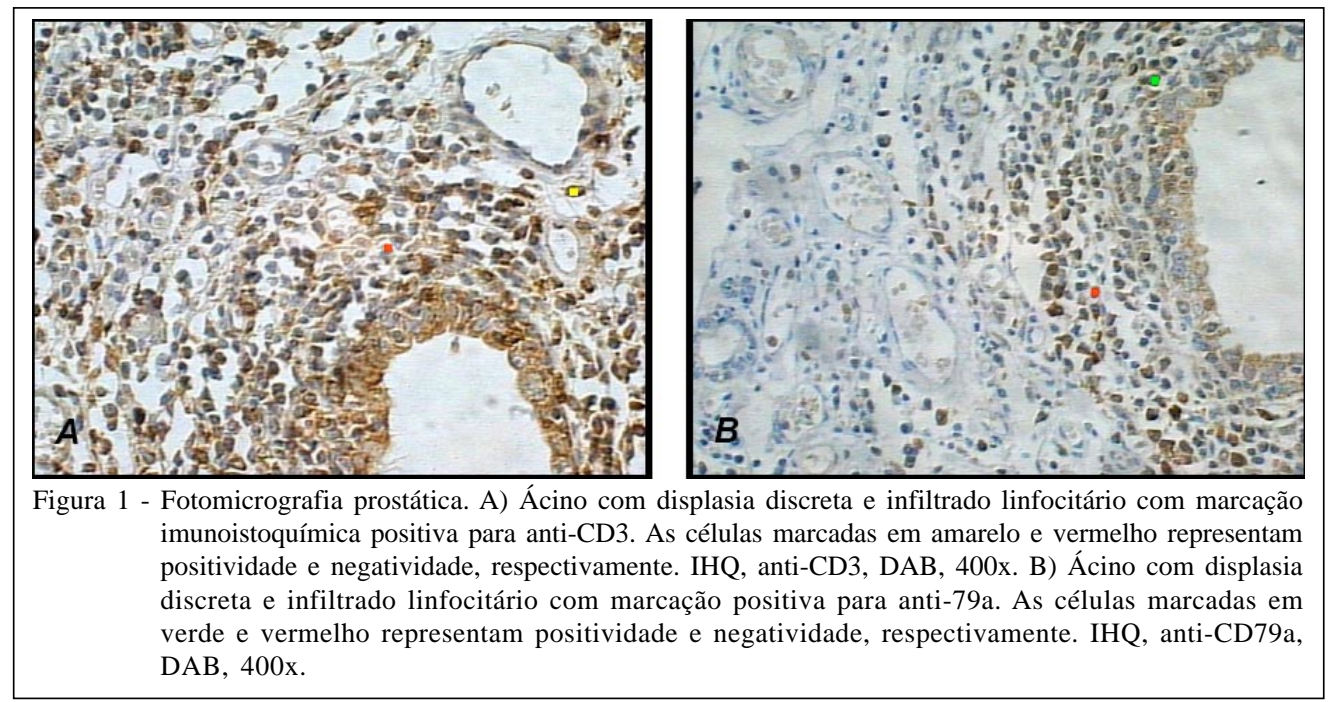

os marcadores utilizados (CD3 e CD79a), bem como a interação entre os efeitos principais grau versus marcador.

\section{RESULTADOS}

Foram catalogadas 42 lesões displásicas, sendo 20 classificadas em discreta (48\%), 16 em moderada (38\%) e seis em acentuada (14\%). Vale ressaltar que o número de lesões excede o número de próstatas, já que estas foram dividas em seis regiões. Assim, foram selecionadas e utilizadas apenas as lâminas referentes às regiões com focos de displasia, sendo descartadas aquelas que não apresentavam a lesão. Apesar disso, não foi observada diferença quanto à distribuição anatômica das displasias nas próstatas estudadas, já que a lesão foi diagnosticada em todas as porções da glândula de forma semelhante. A figura 2

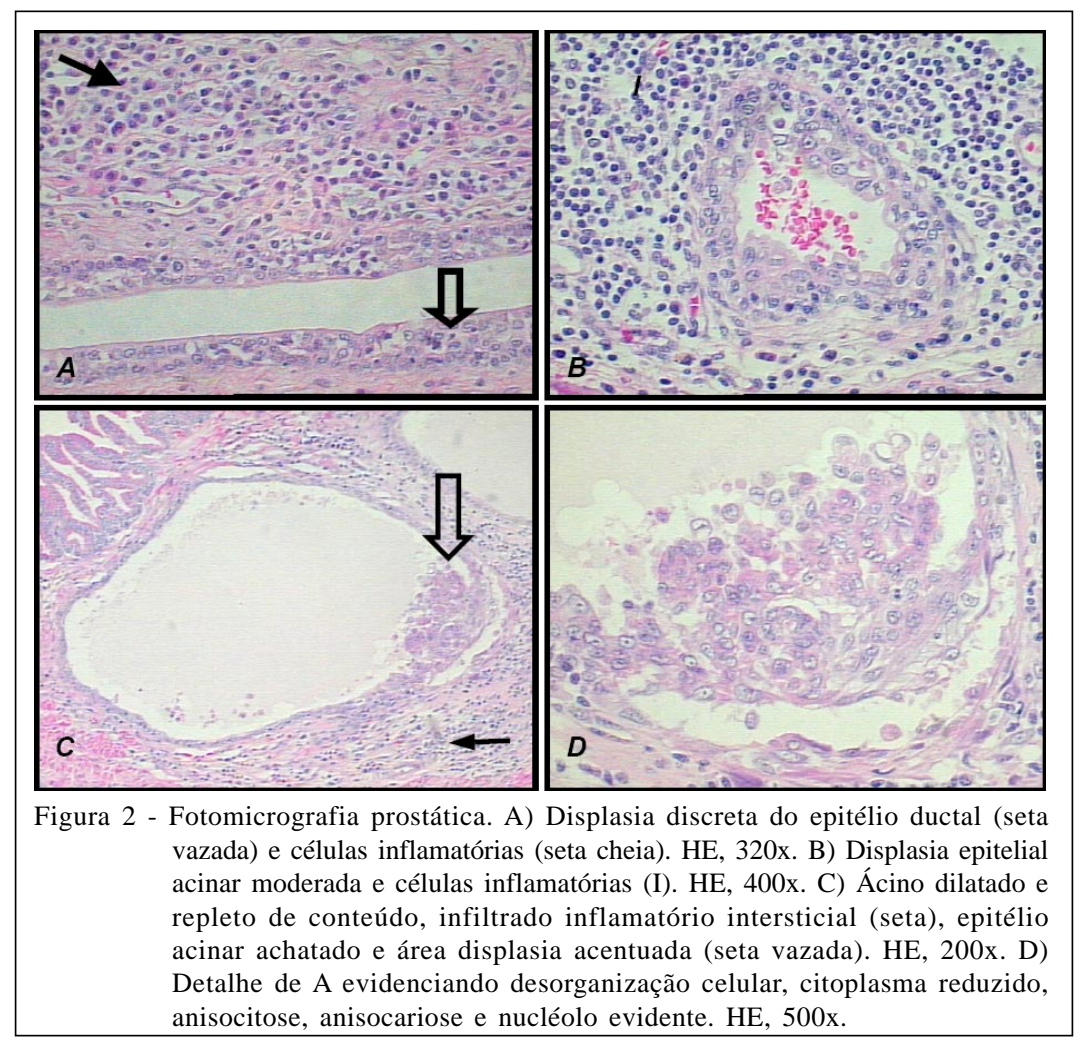

Ciência Rural, v.40, n.5, mai, 2010. 
representa displasia discreta, moderada e acentuada, respectivamente.

Quanto à idade dos animais, observou-se que as lesões displásicas, independente do grau, foram mais frequentes nos cães acima de seis anos, com maior concentração naqueles com idade entre seis e oito anos. Na tabela 1, são apresentados a faixa etária dos animais e o grau de displasia.

Os percentuais de células marcadas para os anticorpos anti-CD3 e anti-CD79a foram de 66 e 30 respectivamente, caracterizando infiltrado linfocitário intersticial com o predomínio de linfócitos T. Vale apontar que a somatória dos percentuais não atinge $100 \%$, pois o fato de as células terem sido contadas em lâminas distintas gera pequenas distorções no número total de células contadas, a despeito da tentativa de escolha de campos semelhantes nas diferentes colorações empregadas. Além disso, existem linfócitos não T/não B e macrófagos, os quais não são imunorreativos aos anticorpos empregados. As estimativas de médias e desvios-padrão mínimos e máximos para o percentual de células marcadas pelos diferentes marcadores são apresentados na tabela 2 .

Observou-se interação entre o grau citohistológico e o marcador utilizado $(\mathrm{P}<0,01)$, indicando dependência dos graus em relação aos marcadores utilizados. As estimativas de médias por graus, para cada marcador avaliado, são apresentadas na tabela 3.

\section{DISCUSSÃO}

KRAWIEC (1994) ressalta que cães inteiros são mais propensos ao desenvolvimento de doença prostática em relação aos castrados. Os casos de displasia observados neste estudo corroboram tal afirmação, visto que as próstatas analisadas são provenientes de animais adultos e sexualmente intactos. Vale destacar que, apesar de a displasia epitelial constituir uma alteração prostática isolada e

Tabela 1 - Idade dos animais em relação ao grau de lesão.

\begin{tabular}{lcccc}
\hline Idade & $\begin{array}{c}\text { Número de } \\
\text { Animais }\end{array}$ & Discreta & Moderada & Acentuada \\
\hline 4 & 1 & 1 & & 1 \\
5 & 1 & 5 & 7 & 3 \\
6 & 4 & 7 & 3 & \\
7 & 4 & 3 & 3 & \\
8 & 2 & 1 & 2 & 2 \\
9 & 1 & & & \\
10 & 1 & 3 & & \\
11 & & &
\end{tabular}

Tabela 2 - Número de observações (N), estimativas de médias (MED) e desvios-padrão (DP) mínimos (MIN) e máximos (MAX) para as percentagens de células marcadas, segundo os marcadores anti-CD3 e antiCD79a.

\begin{tabular}{lccccc}
\hline Marcador & N & MED & DP & MIN & MAX \\
\hline CD3 & 164 & $66 \%$ & 0,12354 & 0,13898 & 0,86037 \\
CD79a & 164 & $30 \%$ & 0,09779 & 0,11714 & 0,74750 \\
\hline
\end{tabular}

em curto prazo não implicar doença clínica, considerase importante a sua interação com outras alterações da glândula, como a presença simultânea de infiltrado intersticial, já que, na próstata humana, essa interação tem sido estudada quanto ao potencial pré-maligno (DE MARZO et al., 2006).

Neste estudo, constatou-se que os graus mais acentuados de displasia são lesões de menor ocorrência, como também ressaltam AMORIM (2001), TESKE et al. (2002) e TERAZAKI (2009). Embora não objeto principal do estudo, observou-se que a hiperplasia prostática benigna e a prostatite foram as lesões mais frequentes da próstata canina, o que também é destacado por TESKE et al. (2002).

No que diz respeito à idade dos cães com displasia prostática, destaca-se que esta esteve presente em um animal com quatro anos e em outro de cinco, sendo o maior número de casos observado naqueles acima de seis anos. Nesse contexto, WATERS \& BOSTWICK (1997a e b) e AQUILINA et al. (1998) descrevem a lesão em animais com idade igual ou superior a sete e seis anos, respectivamente, independente da presença de outras enfermidades prostáticas, sendo imediato sugerir que essa alteração certamente pode ser encontrada em cães adultos e idosos, frequentemente naqueles acima de seis anos. Apesar disso, WATERS \& BOSTWICK (1997a), assim como neste estudo, observaram um animal com displasia em um grupo de cães de um a quatro anos.

Quanto ao infiltrado inflamatório de caráter crônico estudado junto às áreas displásicas do epitélio prostático canino, foi possível verificar, por meio do perfil imunoistoquímico, que este é predominantemente composto por linfócitos T (66\%), caracterizando a imunidade celular como o principal elemento envolvido nesse tipo de reação inflamatória. Esse resultado é semelhante ao de MAHAPOKAI et al. (2001), que obtiveram $80 \%$ de positividade para o anticorpo antiCD3 (marcador linfocitário pan T). Alguma diferença entre os percentuais obtidos nos dois trabalhos pode ser justificada pela intensidade do infiltrado nas regiões avaliadas, visto que no primeiro foram estudadas áreas com discreto, moderado a acentuado infiltrado junto 
Tabela 3 - Interação grau citohistológico da displasia versus marcador imunoistoquímico.

\begin{tabular}{|c|c|c|c|c|}
\hline \multicolumn{5}{|c|}{ Classificação histológica } \\
\hline \multirow{3}{*}{$\begin{array}{l}\text { Marcador } \\
\text { CD3 }\end{array}$} & ------------- & contagens pel & -------------- & --- \\
\hline & Discreta & Moderada & \multicolumn{2}{|c|}{ Acentuada } \\
\hline & $0,63065 \mathrm{C}$ & 0,69839 A & 0,65748 & B \\
\hline CD79a & $0,31820 \mathrm{~A}$ & $0,28356 \mathrm{~B}$ & 0,28275 & $\mathrm{C}$ \\
\hline
\end{tabular}

Médias seguidas pela mesma letra, em uma mesma linha, não diferem entre si pelo teste de Qui-quadrado (P<0,01).

às áreas displásicas, já o segundo envolveu áreas com discreto, moderado e acentuado infiltrado, mas não necessariamente adjacente à displasia. Aliás, o estudo supracitado enfoca o infiltrado em associação com HPB, não fazendo nenhuma menção à displasia, apenas a algumas áreas de atrofia.

É importante enfatizar que não foi encontrada literatura abordando especificamente a interação displasia prostática canina e o infiltrado inflamatório, apenas ORTIZ et al. (1996) descrevem relação significativa entre displasia e infiltrado inflamatório crônico na próstata humana. Contudo, não determinaram o imunofenótipo ou a intensidade do infiltrado, assim como a proximidade com as lesões displásicas.

Em relação ao resultado demonstrando a dependência do grau com o marcador, foi possível interpretar que o número de células T marcadas é maior no infiltrado intersticial próximo aos graus moderado e acentuado de displasia, respectivamente, e a quantidade de células B é maior nas áreas adjacentes à displasia discreta que naquelas próximas às áreas moderada e acentuada. Isso talvez possa refletir o tipo de resposta inflamatória junto às displasias prostáticas caninas, ou seja, inflamação acentuada e celular nos graus moderado e acentuado de displasia, e inflamação discreta e humoral no grau discreto de displasia. Outra hipótese aventada é de que a resposta humoral possa ser mais evidente na fase inicial da displasia, havendo pequena oscilação em queda, com concomitante aumento da resposta celular no processo de evolução da lesão para moderada e acentuada. Vale ressaltar que não foram encontrados dados que reiterem ou desabonem essas hipóteses, apenas MAHAPOKAI et al. (2001), utilizando metodologia imunoistoquímica semelhante, também observaram correlação entre o tipo linfocitário e a intensidade da resposta inflamatória em próstatas caninas com HPB. Contudo, as células não foram contadas, como neste estudo, sendo o infiltrado apenas estimado em discreto, moderado e acentuado, e correlacionado com a intensidade de células marcadas. Além disso, esses mesmos autores afirmam que o desenvolvimento de hiperplasia precede a infiltração inflamatória em cães, situação não confirmada em relação à displasia, sendo certo apenas que estas são frequentemente acompanhadas de infiltrado inflamatório intersticial periacinar de caráter morfológico crônico (DE MOURA, 2004).

Com a finalização deste estudo, algumas questões foram elucidadas, como a confirmação da displasia prostática canina, os graus de intensidade da lesão, a interação com o infiltrado inflamatório e a marcada resposta imune celular. Entretanto, outras explicações serão exaustivamente procuradas no intuito de elucidar a etiopatogenia e o comportamento biológico das lesões displásicas da próstata canina.

\section{CONCLUSÕES}

O infiltrado linfocitário intersticial periacinar junto às áreas de epitélio prostático displásico constituise predominantemente por linfócitos T. Há interação entre o grau histológico da displasia prostática canina e o marcador imunoistoquímico, com oscilação na quantidade de células T e B em função da intensidade da displasia.

\section{AGRADECIMENTO}

Fundação de Amparo à Pesquisa do Estado de São Paulo - FAPESP (auxílio à pesquisa 04/06052-3).

\section{REFERÊNCIAS}

AMORIM, R.L. Estudo clínico, laboratorial e anatomopatológico das próstatas de cães adultos. 2001. $117 \mathrm{f}$. Tese (Doutorado) - Faculdade de Medicina Veterinária e Zootecnia, Universidade Estadual Paulista (UNESP), Botucatu, SP.

AQUILINA, J.W. et al. High grade prostatic intraepithelial neoplasia in military working dogs with and without prostate cancer. Prostate, v.36, p.189-193, 1998. Disponível em: <http:// www3.interscience.wiley.com/cgi-bin/fulltext/34418/PDFSTART>. Acesso em: 25 mar. 2010. doi: 10.1002/(SICI)10970045(19980801)36:3<189::AID-PROS7>3.0.CO;2-C.

BRASILEIRO FILHO, G. et al. Distúrbios do crescimento e da diferenciação cellular. In: BRASILEIRO FILHO, G. Bogliolo 
patologia. 7.ed. Rio de Janeiro: Guanabara Koogan, 2006. p.175-236.

DE MARZO, A.M. et al. A working group classification of focal prostate atrophy lesions. Am J Surg Pathol, v.30, n.10, p.1281-1291, 2006. Disponível em: <http:// o v i d s p.t x. o v i d. c o m/s p - 2.3.1/ ovidweb.cgi? WebLinkFrameset $=1 \& S=$ KNPFFPMEAOD

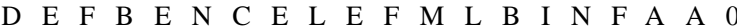
$0 \&$ returnUrl=http $\% 3 a \% 2$ f\%2fovidsp.tx.ovid.com $\% 2$ fsp2 . $3.1 \% 2 \mathrm{f} \mathrm{ovid} \mathrm{w} \mathrm{e} \mathrm{b.c} \mathrm{g} \mathrm{i} \mathrm{\%} 3$ f \% $26 \mathrm{~F} \mathrm{u} \mathrm{l} \mathrm{l} \mathrm{\%} 2$ bText\%3dL\%257cS.sh.15.16\%257c0\%257c00000478200610000-00011\%26S\%3dKNPFFPMEAODDEFBENC ELEFMLBINFAA00\&directlink=http \%3a\%2f\%2fgraph ics.tx.ovid.com $\% 2$ fovftpdfs $\% 2$ fFPDDNCMLEFBEAO 00\%2ffs047\%2fovft\%2flive\%2fgv024\%2f00000478\%2f00000478200610000 - 00011 . pd f \& fil en a m e = A + Workin g+Group+Classification+of+Focal+Prostate+Atrophy+Lesions $>$. Acesso em: 25 mar. 2010. doi: 10.1097/01.pas.0000213289.50660.be.

DE MOURA, V.M.B.D. Estudo laboratorial, anatomopatológico e imunoistoquímico da próstata de cães adultos. 2004. 144f. Tese (Doutorado em Patologia Animal) - Faculdade de Medicina Veterinária e Zootecnia, Universidade Estadual Paulista (UNESP) Botucatu, SP.

KRAWIEC, D.R. Canine prostate disease. J Am Vet Med Assoc, v.204, n.10, p.1561-1564, 1994.

LEE, C. et al. Intrinsic and extrinsic factors controlling benign prostatic growth. Prostate, v.31, p.131-138, 1997. Disponível em: <http://www3.interscience.wiley.com/cgi-bin/fulltext/ 49108/PDFSTART>. Acesso em: 25 mar. 2010. doi: 10.1002/ ( S I C I ) 1097 - 0045 ( 19970501 ) 31 : 2<131:: A I D PROS9>3.0.CO;2-Q.

MAHAPOKAI, W. et al. Immune response in hormonallyinduced prostatic hyperplasia in the dog. Vet immunol Immunopathol, v.78, p.297-303, 2001. Disponível em: $<\mathrm{h} \mathrm{t} \mathrm{t} \mathrm{p} \mathrm{:} \mathrm{/} \mathrm{/} \mathrm{w} \mathrm{w} \mathrm{w.} \mathrm{s} \mathrm{c} \mathrm{i} \mathrm{e} \mathrm{n} \mathrm{c} \mathrm{ed} \mathrm{i} \mathrm{re} \mathrm{c} \mathrm{t.} \mathrm{c} \mathrm{o} \mathrm{m/}$ science? ob=MImg\& imagekey=B6TD5-42PBYFW-6$3 \&$ \&_di $=5189 \&$ \&_user $=686368 \&$ \&_pi $=$ S 01652427 $01002367 \&$ orig =search \&_coverDate $=02 \% 2 \mathrm{~F}$ 10\%2F2001\&_sk=999219996\&view $=$ c \&wchp $=$ dGLzVtz-
zSkzk\&md5=6a70781f3e5a4daf610b1bcf19eb5da0\&ie =/ sdarticle.pdf $>$. Acesso em: 25 mar. 2010. doi:10.1016/S01652427(01)00236-7.

SAS. User's guide: basic and statistic. Cary, 1995. 1686p.

ORTIZ, J.L.M. et al. Lesiones premalignas y otras benignas en nódulos de hiperplasia adenomatosa. Arch Esp Urol, v.49, p.684-687, 1996.

TESKE, E. et al. Canine prostate carcinoma: epidemiological evidence of an increases risk in castrated dogs. Molecular and cellular endocrinology, v.197, n.1-2 p.251-255, 2002. Disponível em: $<\mathrm{h} \mathrm{t} \mathrm{t} \mathrm{p} \mathrm{:} \mathrm{/} \mathrm{/} \mathrm{w} \mathrm{w} \mathrm{w.} \mathrm{s} \mathrm{c} \mathrm{i} \mathrm{e} \mathrm{n} \mathrm{c} \mathrm{e} \mathrm{d} \mathrm{i} \mathrm{r} \mathrm{e} \mathrm{c} \mathrm{t} \mathrm{.} \mathrm{c} \mathrm{o} \mathrm{m/}$ science?_ob=MImg\&_imagekey=B6T3G-475GT1Y-C3\&_cdi $=4946 \&$ \&_user $=686368 \&$ \& pii $=$ S 030372070200 $2617 \&$ \& orig=search \&_coverDate $=11 \% 2$ F $29 \% 2$ F 2 $002 \&$ \& k $=998029998 \&$ view $=$ c \& w chp $=$ d G L z V tb zSkWb\&md5=65458fb4a8512e3a24a0ac0d258283c8\&ie=/ sdarticle.pdf >. Acesso em: 25 março 2010. Acesso em: 25 mar. 2010. doi:10.1016/S0303-7207(02)00261-7.

TERAZAKI, P.M. Caracterização da próstata canina quanto a aspectos evolutivos na evolução para o carcinoma prostático. 2009. 109f. Tese (Doutorado em Patologia Experimental e Comparada) - Faculdade de Medicina Veterinária e Zootecnia, Universidade de São Paulo (USP), SP

WATERS, D.J.; BOSTWICK, D.G. The canine is a spontaneous model of intraepithelial neoplasia and prostate cancer progression. Antic Res, v.17, p.1467-1470, 1997a.

WATERS, D.J.; BOSTWICK, D.G. Prostatic intraepithelial neoplasia occurs spontaneously in the canine prostate. J Urol, v.157, p.713-716, 1997b. Disponível em: <http:// $\mathrm{w}$ w $\mathrm{w}$. $\mathrm{s}$ c i e $\mathrm{n}$ c e d i $\mathrm{r}$ e $\mathrm{c}$. c o m / science?_ob=MImg\&_imagekey=B7XMT-4HM6P25-3B$1 \&$ \&_di $=29679 \&$ \&_user $=686368 \&$ \& pii $=$ S 002253470 165256 X \&_orig = search \&_cover Date $=02 \%$ 2F28\%2F1997\&_sk=998429997\&view $=$ c\&wchp $=$ dGLzVtzzSkWb\&md5=050f40e26c6b30a6e17a9b76787821f1\&ie=/ sdarticle.pdf>. Acesso em: 25 mar. 2010. doi:10.1016/S00225347(01)65256-X. 